Bulletin of the Section of Logic

Volume 46:3/4 (2017), pp. 151-168

http://dx.doi.org/10.18778/0138-0680.46.3.4.01

Tomasz Jarmużek*, Mateusz Klonowski ${ }^{\dagger}$ and Jacek Malinowski

\title{
BAYESIAN PROPOSITIONAL LOGIC
}

\begin{abstract}
We define and investigate from a logical point of view a family of consequence relations defined in probabilistic terms. We call them relations of supporting, and write: $\mathbb{F}_{w}$, where $w$ is a probability function on a Boolean language. $A \mathbb{F}_{w} B$ iff the fact that $A$ is the case does not decrease a probability of being $B$ the case. Finally, we examine the intersection of $\approx_{w}$, for all $w$, and give some formal properties of it.
\end{abstract}

Keywords: logical entailment, statistical inference, Bayesian inference, corroboration, confirmation.

\section{Introduction}

Let us consider a popular example concerning conditional probability. Suppose there are two bowls full of cookies. Bowl 1 contains 10 chocolate chips and 30 plain cookies, while bowl 2 contains 20 of each. We pick a bowl at random, and then pick a cookie at random. The cookie turns out to be a plain one. How probable is it that we picked it out of bowl 1? Intuitively, it seems clear that the answer should be more than a half, since there are more plain cookies in bowl 1. The precise answer is given by Bayes' formula:

* The part of research made by Tomasz Jarmużek presented in the following article was financed by National Science Centre, Poland, number of grant: 2015/19/B/HS1/02478.

$\dagger$ The part of research made by Mateusz Klonowski presented in the following article was financed by National Science Centre, Poland, number of grant: 2015/19/N/HS1/02401. 


$$
p(H \mid O)=\frac{p(O \mid H) \cdot p(H)}{p(O)}
$$

where:

- $p(O \mid H)$ is the conditional probability of seeing the observation $O$ given that the hypothesis $H$ is true

- $p(H)$ is the prior probability of $H$

- $p(O)$ is the prior probability of $O$.

Now let $H_{1}$ corresponds to bowl 1 , and $H_{2}$ to bowl 2. It is given that the bowls are identical, thus $p\left(H_{1}\right)=p\left(H_{2}\right)$, and the two must add up to 1 , so both are equal to 0,5 . The datum $O$ is the observation of a plain cookie. From the content of the bowls, we know that $p\left(O \mid H_{1}\right)=30 / 40=0,75$ and $p\left(O \mid H_{2}\right)=20 / 40=0,5$. Moreover we know that $p(O)=50 / 80=0,625$, because there are 50 plain cookies among 80 cookies in general. More precise $p(O)=p\left(O \mid H_{1}\right) \cdot p\left(H_{1}\right)+p\left(O \mid H_{2}\right) \cdot p\left(H_{2}\right)=(3 / 4) \cdot(1 / 2)+$ $(1 / 2) \cdot(1 / 2)=0,625$. Bayes' formula then yields $p\left(H_{1} \mid O\right)=0,6$. Before observing the cookie, the probability bowl 1 has been chosen, was 0,5. After observing the cookie is a plain one, we revise the probability to $p\left(H_{1} \mid O\right)$, which is 0,6 .

The hypothesis $H_{1}$ has been here tested by the evidence $O$. The relation between $O$ and $H_{1}$ seems to be similar to the relation between a premiss and a conclusion.

The above procedure of testing hypotheses by evidence has been extensively studied by philosophers of science as one of possible answers to the question of how we can evaluate a degree to which available experiments and observations support a general scientific hypothesis. For more details we refer to Carnap's confirmation theory elaborated in [1] and to corroboration theory proposed in the central book in this area [10]. For the review of more recent developments we refer the reader to [2], [5] and [6].

The aim of this paper is to investigate from a purely logical point of view the relation described above. We define a propositional language which allows us to consider events like $O$ and $H$ as sentences. Then we construct some semantics by considering probabilities as valuations. This enables us to determine relation of support $\approx$. 


\section{Basic concepts}

Let $L_{n}$ denotes a $n$-generated sentential language with connectives $\wedge, \vee$, $\neg, \rightarrow, \leftrightarrow$ generated by $n$-element set $V=\left\{q_{1}, \ldots q_{n}\right\}$ of sentential variables. We will identify language $L_{n}$ with its set of sentences (well formed formulas). By small characters $p, q, r$ (with or without indices) we will denote the sentential variables while capitals $A, B, C, P, Q, R$ denote arbitrary sentences of $L_{n}$.

By classical logic in the language $L_{n}$ we mean the binary relation $\models^{n}$ between sets of sentences and single sentences defined in the following standard way: $X \models^{n} A$ iff for every classical valuation $v: L_{n} \longmapsto\{0,1\}$, $v(A)=1$, whenever $v(X) \subseteq\{1\}$. Symbol $\models^{n} A$ means that $A$ is a classical tautology. If it does not lead to misunderstanding we will skip superscript $n$ in $\models^{n}$.

Relation $\models$ forms here a base for another inference-like relations defined by means of probability functions. The general framework in which we work is that of [7].

Instead of a pure probability we use here a notion of probabilistic valuation. Our definition is based on well known Kolmogorov's axiomatization of a finitely additive probability function (probability measure) presented in [3].

By a probabilistic valuation on the language $L_{n}$ we mean a function defined on $L_{n}$ with values in the unit interval of reals $[0,1]$. Such function $w$, for each sentences $A, B$ satisfies the following conditions:

(W1) $0 \leqslant w(A) \leqslant 1$

(W2) $w(A)=1$, for some sentence $A$

(W3) $w(A) \leqslant w(B)$, whenever $A \models B$

(W4) $w(A \vee B)=w(A)+w(B)$, whenever $A \models \neg B$.

The definition was - among others - introduced in [7, p. 113].

The conditions (W1)-(W4) correspond to Kolomogorov's axioms defining a finitely additive probability function. For many aims it is necessary to strength (W4) to allow infinite joins. For example, it would be needed, if we considered infinitely generated sentential language - i.e. a language with infinitely many sentential variables. However, in this paper we consider only finitely generated sentential languages and we do not need infinite joins of sentences. 
All classical valuations satisfy the conditions (W1)-(W4) and so they can be recognised as a limit case of probabilistic valuations. Of course not every probabilistic valuation is a classical one.

We omit the adjective 'probabilistic' in the rest of this paper. Henceforth a 'valuation' means always 'probabilistic valuation'. Furthermore, we assume an abbreviation, by writing $A \models B$, we shortly declare that $A \models B$ and $B \models A$. Sometimes, we also will write CPL, having in mind classical propositional logic.

Next proposition describes some well known properties of probabilistic valuation:

Proposition 1. For any valuation $w$ the following condition holds:

(W5) $w(A)=1$, if $\models A$

(W6) $w(\neg A)=1-w(A)$

(W7) $w\left(A_{1} \vee \ldots \vee A_{n}\right)=w\left(A_{1}\right)+\ldots+w\left(A_{n}\right)$, whenever $A_{i} \models \neg A_{j}$, for all $i \neq j$ such that $1 \leqslant i, j \leqslant n$

(W8) $w(A)=w(B)$, if $A \models B$

(W9) $w(A \vee B)=w(A)+w(B)-w(A \wedge B)$.

Most of those conditions for probabilistic valuations were considered by Makinson in [7]. For example, a proof of (W5) is given on page 191, while some proofs of (W6) and (W8) on page 192.

It is well known that any function $f:\left\{q_{1}, \ldots, q_{n}\right\} \longmapsto\{0,1\}$ can be uniquely extended to the classical valuation of $L_{n}$. However in case of probabilistic valuations it is more complicated. A function $f:\left\{q_{1}, \ldots, q_{n}\right\} \longmapsto$ $[0,1]$ might have many possible extensions to a probabilistic valuation.

By a literal of $L_{n}$ we mean either a sentential variable or a negation of sentential variable. By a state description in the language $L_{n}$ we mean any conjunction of $n$ literals in fixed order. Each $i$-th conjunct of a such conjunction is either $q_{i}$ or $\neg q_{i}$. Of course in any language $L_{n}$ there is exactly $2^{n}$ state descriptions. A function $f$ from the set SD of all of $2^{n}$ state descriptions into the unit interval $[0,1]$ such that $\sum_{s \in \mathrm{SD}} f(s)=1$ will be called a probability distribution.

The following proposition holds by classical propositional logic.

Proposition 2. Every sentence which is not a contradiction is classically equivalent to a disjunction of unique non-empty subset of state descriptions. 
In the further parts of our paper when we take for a sentence $A$ some logically equivalent disjunction of state descriptions $s_{1} \vee \cdots \vee s_{n}$, we always assume that $\left\{s_{1}, \ldots, s_{n}\right\}$ is a unique non-empty subset of state descriptions mentioned in proposition 2 .

THEOREM 3. Each probability distribution can be in a unique way extended to a valuation satisfying (W1)-(W4).

Proof. Let sentence $A$ of language $L_{n}$ be not a contradiction. By proposition 2 we take a disjunction of state descriptions $s_{1}, \ldots, s_{k}$ classically equivalent to $A$. Then we put $w(A)=f\left(s_{1}\right)+\ldots+f\left(s_{k}\right)$. If $A$ is a contradiction, then we put $w(A)=0$. It is easy to check that $w$ satisfies conditions (W1)-(W4). The idea of probability distribution and outline of the proofs that concerns can be also found in [7, p. 115-116].

Theorem 3 shows that there is one-to-one correspondence between valuations and probability distributions, since state of descriptions are always uniquely valuated, valuations satisfy conditions (W1)-(W4), and then we can reduce any valuation $w$ to an probability distribution $f$.

For this reason probability distributions give us a formal tool for the study of probabilistic inference relation defined below.

Let $w$ denote a probabilistic valuation. Then for any sentences $A$ and $B$ such that $w(A) \neq 0$ a function $w_{A}(B)$ defined as

$$
w_{A}(B)=\frac{w(A \wedge B)}{w(A)}
$$

will be called a conditional probability.

Writing a probability function, we will always assume that a given denominator of the fraction is different from zero. Next well known theorem express an important property of conditional probability:

Bayes' Theorem. For any sentences $A, B$ :

$$
w_{A}(B)=\frac{w_{B}(A) \cdot w(B)}{w(A)}
$$

Makinson in [7] mentioned certain families of probabilistic consequence relations. Among them he distinguished some relations that are very similar to what we will examine here. He called the relations incremental probabilistic consequences, but did not study their properties (except the failure 
of monotonicity) [7, p. 130]. Some examination of those interpretations we leave as a subject of a future work.

Now we come to the main notion of this paper - probabilistic entailment. Let $A, B \in L_{n}$. We say that $A$ supports $B$ under a valuation $w$, in symbols $A \approx_{w}^{n} B$ if and only if either $w(A)=0$ or $w(A) \neq 0$ and $w_{A}(B) \geqslant w(B)$. The fact that $A \approx_{w}^{n} B$ for a valuation $w$ in $L_{n}$ will be denoted by $A \approx_{w} B$, if the context clearly indicates the language $L_{n}$. The fact that $A \approx_{w}^{n} B$ for all valuation $w$ will be denoted by $A \approx^{n} B$. Obviously $\approx^{n} \subseteq \approx_{w}^{n}$. If the context clearly indicates the language $L_{n}$, then we simply write $A \approx^{n} B$. Probability theorists often examined and used relations $\approx_{w}$, but so far they have not made any comprehensive study of their logical properties.

The relation of supporting is a kind of statistical or probabilistic entailment. However it is better to speak about supporting relation rather than about some kind of logical entailment, since such relation does not satisfy the main feature of logical entailment, i.e. it does not guarantee that conclusions are true, whenever premisses are true. Instead it has got a weaker feature: the level of certainty of conclusion does not decrease, if premisses are true.

On the other hand the relation of supporting generalizes logical entailment and seems to satisfy natural intuitions. Thus suppose that $A$ and $B$ describe some events and if the event described by $A$ is the case, then the event described by $B$ is not less probable and maybe even more probable than without $A$. By increasing probability, in fact, we understand that $B$ can be more probable with $A$ than without $A$. That is way we call $\approx$ supporting relation.

Bayes' theorem allows us to characterize the relation of $\approx$ in terms of a valuation $w$. Theorem 4 below summarizes these results. What is here especially unexpected, is that conditions (i) and (iv) are equivalent, which shows that the relation of supporting is symmetric. Although such equivalence is just an obvious consequence of the definition of $\approx$ it seems to be an important feature which distinguishes statistical reasoning from other kinds of reasoning.

TheOREM 4. The following conditions are equivalent:

(i) A supports $B$ under $w$

(ii) $w(A \wedge B) \cdot w(\neg B) \geqslant w(A \wedge \neg B) \cdot w(B)$

(iii) $w(A \wedge B) \geqslant w(A) \cdot w(B)$

(iv) $B$ supports $A$ under $w$. 
Proof. We will prove equivalence of (ii) and (iii).

1. $w(A \wedge B) \geqslant w(A) \cdot w(B)$ iff by (W8)

2. $w(A \wedge B) \geqslant w((A \wedge \neg B) \vee(A \wedge B)) \cdot w(B)$ iff

3. $w(A \wedge B) \geqslant(w(A \wedge \neg B)+w(A \wedge B)) \cdot w(B)$ iff

4. $w(A \wedge B)-w(A \wedge B) \cdot w(B) \geqslant w(A \wedge \neg B) \cdot w(B)$ iff

5. $w(A \wedge B) \cdot(1-w(B)) \geqslant w(A \wedge \neg B) \cdot w(B)$ iff

6. $w(A \wedge B) \cdot w(\neg B) \geqslant w(A \wedge \neg B) \cdot w(B)$.

The remaining claims are immediate consequences of Bayes' theorem and the definition of $\approx$.

\section{Characterization of $\approx$}

In this section we consider the relations $\approx^{n}$ for some $n$-generated sentential language.

S. Kraus, D. Lehmann and M. Magidor considered in [4] some Gentzen style rules as an axiomatization of certain classes of non-monotonic supraclassical logic.

$$
\frac{\models A \leftrightarrow B, \quad A \approx C}{B \approx C}
$$$$
\frac{\models A \leftrightarrow B, \quad C \approx A}{C \approx B}
$$

$$
\frac{A \models B}{A \approx B}
$$

(Ctp)

$$
\frac{A \approx B}{\neg B \approx \neg A}
$$

We will add two more rules to the list they proposed and then we check all of them for relation $\approx$.

(Sym)

$$
\frac{A \approx B}{B \approx A}
$$

(Sim)

$$
\frac{A \wedge C \approx B \quad A \wedge \neg C \approx B}{A \approx B}
$$

Theorem 5. The rules (LLE), (RLE), (Cl), (Sim), (Sym), (Ctp) hold for any valuation. 
Proof. (LLE) Suppose that $\models A \leftrightarrow B$ and $A \approx C$, then $w(B)=w(A)$ and hence $w(B \wedge C)=w(A \wedge C) \geqslant w(A) \cdot w(C)=w(B) \cdot w(C)$. So, $B \approx C$. Hence by Theorem $4, B \approx C$.

(RLE) Suppose that $=A \leftrightarrow B$ and $C \approx A$. Then $w(B)=w(A)$. Moreover, $w(C \wedge B)=w(C \wedge A) \geqslant w(C) \cdot w(A)=w(C) \cdot w(B)$. Hence by Theorem $4, C \approx B$.

(Cl) Suppose that $A \models B$, then $A \models A \wedge B$. Therefore by (W8), $w(A)=$ $w(A \wedge B)$. If $w(A)=0$, then by definition of $\approx, A \approx B$. Let $w(A) \neq$ 0 . Hence $w_{A}(B)=\frac{w(A \wedge B)}{w(A)}=1$, and so $w(B) \leqslant w_{A}(B)$. Therefore by Theorem $4, A \approx B$.

(Ctp) Suppose that $A \approx B$, then by Theorem $4, w(A \wedge B) \geqslant w(A) \cdot w(B)$.

1. $w(\neg A \wedge \neg B)=1-w(A \vee B)$

by CPL, (W6)

2. $1-w(A \vee B)=1-w(A)-w(B)+w(A \wedge B)$ by (W9)

3. $1-w(A)-w(B)+w(A \wedge B) \geqslant 1-w(A)-w(B)+w(A) \cdot w(B)$ by assumptions

4. $1-w(A)-w(B)+w(A) \cdot w(B)=(1-w(A)) \cdot(1-w(B))$

5. $(1-w(A)) \cdot(1-w(B))=w(\neg A) \cdot w(\neg B)$ by (W6)

6. $w(\neg A \wedge \neg B) \geqslant w(\neg A) \cdot w(\neg B)$ 1,5 .

Hence, by Theorem $4, \neg A \approx \neg B$.

(Sym) The rule (Sym) is an obvious consequence of Theorem 4 .

(Sim) Suppose that $A \wedge C \approx B$ and $A \wedge \neg C \approx B$. Therefore by Theorem 4, $w(A \wedge C \wedge B) \geqslant w(A \wedge C) \cdot w(B)$ and $w(A \wedge \neg C \wedge B) \geqslant w(A \wedge \neg C) \cdot w(B)$.

1. $w(A \wedge B)=w((A \wedge B \wedge C) \vee(A \wedge B \wedge \neg C)) \quad$ by CPL and (W8)

2. $w((A \wedge B \wedge C) \vee(A \wedge B \wedge \neg C))=w(A \wedge B \wedge C)+w(A \wedge B \wedge \neg C)$ by $\mathrm{CPL}$ and (W4)

3. $w(A \wedge B \wedge C)+w(A \wedge B \wedge \neg C) \geqslant w(A \wedge C) \cdot w(B)+w(A \wedge \neg C) \cdot w(B)$ by assumptions

4. $w(A \wedge C) \cdot w(B)+w(A \wedge \neg C) \cdot w(B)=w(B) \cdot(w(A \wedge C)+w(A \wedge \neg C))$

5. $w(B) \cdot(w(A \wedge C)+w(A \wedge \neg C))=w(B) \cdot w((A \wedge C) \vee(A \wedge \neg C))$ by $\mathrm{CPL}$ and (W4)

6. $w(B) \cdot w((A \wedge C) \vee(A \wedge \neg C))=w(A) \cdot w(B) \quad$ by CPL and (W8)

7. $w(A \wedge B) \geqslant w(A) \cdot w(B)$ $1,6$.

So, by Theorem $4, A \approx B$. 
According to $(\mathrm{Cl})$ classical consequence relation is included in $\approx$. Theorem 5 shows that $\approx$ is essentially "larger" than $\models$, since $\approx$ fulfils (Sym).

Theorem 6. (David Makinson) $A \approx B$ iff either $A \models B$ or $B \models A{ }^{1}$

Proof. $\Longleftarrow$ part is an immediate consequence of $(\mathrm{Cl})$ and $(\mathrm{Sym})$. To prove $\Longrightarrow$ let us suppose that $A \not \models B$ and $B \not \models A$. By CPL it means that there are two distinct state descriptions, sentences $s^{\prime}$ and $s^{\prime \prime}$ such that $s^{\prime} \models A, s^{\prime} \models \neg B, s^{\prime \prime} \models B$ and $s^{\prime \prime} \models \neg A$.

Let $r$ denote any real number $0<r<1$. Let us define the probability distribution $f$ on the set SD of state descriptions in the following way:

$$
f(s)= \begin{cases}r & \text { if } s=s^{\prime} \\ 1-r & \text { if } s=s^{\prime \prime} \\ 0 & \text { otherwise }\end{cases}
$$

By Theorem 3 function $f$ can be uniquely extended to valuation $w$. By proposition 2 we have such unique set of state descriptions $\left\{s_{1}, \ldots, s_{k}\right\}$ that $A \wedge B \models s_{1} \vee \ldots \vee s_{k}$. Surely, neither $s^{\prime} \in\left\{s_{1}, \ldots, s_{k}\right\}$, nor $s^{\prime \prime} \in\left\{s_{1}, \ldots, s_{k}\right\}$. So, by definition of extension of $f$-given in Theorem $3-w(A \wedge B)=0$.

By proposition 2 we have also such unique set of state descriptions $\left\{\bar{s}_{1}, \ldots, \bar{s}_{k}\right\}$ that $B \models \bar{s}_{1} \vee \ldots \vee \bar{s}_{k}$. Surely, $s^{\prime} \notin\left\{\bar{s}_{1}, \ldots, \bar{s}_{k}\right\}$, but $s^{\prime \prime} \in$ $\left\{\bar{s}_{1}, \ldots, \bar{s}_{k}\right\}$. So, by definition of extension of $f, w(B)=1-r>0$.

Combining two above facts, we get $w(A \wedge B)<w(B)$, and so $A \not \notin B$.

From Theorem 6 we have an obvious corollary.

Corollary 1. $A \approx B$ iff either $\models A \rightarrow B$ or $\models B \rightarrow A$.

Similarly as for $\models$ we can define a notion of tautology for $\approx$ relation as a sentence which is supported by any sentence. It is a natural question whether there are non-classical tautologies of $\approx$. The answer appears to be unexpected.

TheOREM 7. The following conditions are equivalent:

(i) A is a classical tautology or a contradiction.

(ii) For any $B, B \approx A$.

(iii) For any $B, A \approx B$.

${ }^{1}$ This theorem is by David Makinson. It was communicated in an email letter. 
Proof. Ad. (i) $\Longrightarrow$ (ii). Suppose that $A$ is a classical tautology. Then, because any function $w$ is an extension of some probability distribution, $w(A)=1$. Since we have $A \wedge B \models B$, then by (W8) $w(A \wedge B)=w(B)$. Therefore $w(B \wedge A)=w(B)=w(A) \cdot w(B)$. Hence by Theorem $4, B \approx A$.

Suppose that $A$ is a contradiction. Then, since any function $w$ is an extension of some probability distribution, $w(A)=0$. So we have $w(A \wedge$ $B) \geqslant 0=w(A) \cdot w(B)$. Hence by Theorem $4, B \approx A$.

Ad. (ii) $\Longrightarrow$ (iii). It follows from the rule (Sym).

Ad. (iii) $\Longrightarrow$ (i). Suppose that $A$ is neither a classical tautology, nor a contradiction. We will construct a probability valuation $\bar{w}$ as an extension of some probability distribution with $\bar{w}(A)=1 / 2$ and $\bar{w}(A \wedge \neg A)=0<$ $1 / 4=1 / 2 \cdot 1 / 2=\bar{w}(A) \cdot \bar{w}(\neg A)$. Then by Theorem $4, A \not \neg A$.

Let us recall that $A$ is a sentence in some $n$-generated language, so in such a language there are $2^{n}$ state descriptions. By proposition $2 A$ is classically equivalent to some disjunction $B$ of a unique non-empty subset of state descriptions. Let $S_{B}$ denote the set of all state descriptions occurring in $B$. Suppose that $S_{B}$ has got $k$ elements. We define a function $\bar{f}$ on SD in the following way:

$$
\bar{f}(s)= \begin{cases}\frac{1}{2 k} & \text { if } s \in S_{B} \\ \frac{1}{2\left(2^{n}-k\right)} & \text { if } s \notin S_{B} .\end{cases}
$$

It is to check that:

$$
\sum_{s \in \mathrm{SD}} \bar{f}(s)=\sum_{i=1}^{k} \frac{1}{2 k}+\sum_{i=1}^{2^{n}-k} \frac{1}{2\left(2^{n}-k\right)}=1 .
$$

Therefore $\bar{f}$ is a probability distribution. By Theorem $3 \bar{f}$ can be uniquely extended to valuation $\bar{w}: L_{n} \mapsto[0,1]$. So we have:

$$
\bar{w}(B)=\sum_{s \in S_{B}} \bar{f}(s)=\sum_{i=1}^{k} \frac{1}{2 k}=1 / 2 .
$$

So by (W6), $\bar{w}(\neg B)=1-w(B)=1 / 2$. By (W8), since $A \models B$ and $\neg A \models \neg B, \bar{w}(A)=\bar{w}(B)$ and $\bar{w}(\neg A)=\bar{w}(\neg B)$. Therefore $\bar{w}(A)=1 / 2=$ $\bar{w}(\neg A)$. 
The proof could be simplified in the way avoiding the above calculations. Such a simplified proof uses Theorem 6 for the last implication. However, we have decided to present here all that redundant calculations to show the complete construction of a valuation. Let us note that for any contingent sentence $A$ and a real number $0<a<1$ we can construct in a way similar to that in the proof such a valuation $w$ that $w(A)=a$.

By $\sim^{n}$ we denote the least binary relation on $L_{n}$ closed with respect to $(\mathrm{Cl})$ and $(\mathrm{Sym})$.

Proposition 8. (Representation) For any $n, \boldsymbol{\sim}^{n}=\mathbb{}^{n}$.

Proof. From Theorem 6 it is enough to show that $A \sim B$ if and only if $A \models B$ or $B \models A$.

$\Longleftarrow$ If $A \models B$, then by $(\mathrm{Cl}) A \sim B$. If $B \models A$, then by $(\mathrm{Cl}) B \sim A$ and then by $(\mathrm{Sym}) A \sim B$.

$\Longrightarrow$ If $A \sim B$, then by (Sym) $B \sim A$. However, $\sim$ is closed under $(\mathrm{Cl})$, so we can get $A \models B$ or $B \models A$.

\section{Some properties of $\approx_{w}$}

In this section we consider the relations $\approx_{w}^{n}$ for arbitrary valuation $w$ and some $n$-generated sentential language.

We have a set of additional rules that may characterize a consequence relation:

(Mon)

$$
\frac{\models A \rightarrow B, \quad C \approx A}{C \approx B}
$$

$$
\frac{A \wedge B \approx C, \quad A \approx B}{A \approx C}
$$$$
\frac{A \approx C, \quad B \approx C}{A \wedge B \approx C}
$$

$$
\frac{A \approx C}{A \wedge B \approx C}
$$

$$
\frac{A \approx B, \quad A \approx C}{A \wedge B \approx C}
$$




$$
\frac{A \approx B, \quad B \approx A, \quad A \approx C}{B \approx C}
$$$$
\frac{A \approx C, \quad B \approx C}{A \vee B \approx C}
$$

$$
\frac{A \approx B, \quad B \approx C}{A \approx C}
$$

As a consequence of Theorem 6 the relation $\approx$ is closed under the rules (Ad) and (Or). However, the rest of the rules ((RW), (Cut), (Mon), (CM), (Eq), (Ad), (T)) fails.

For example, to show the failure of (CM), we take $p \approx p \wedge q$ and $p \approx p \wedge r$, since $p \wedge q \models p$ and $p \wedge r \models p$ (by (Cl) and Theorem 6). However, at the same time $p \wedge(p \wedge q) \not p \wedge r$. Other cases have similar counterexamples.

The next theorem, Theorem 9, shows that (Ad) and (Or) are not the case for some $\approx_{w}^{n}$. As a consequence, $\approx^{n} \subset \approx_{w}^{n}$, for some $w$.

TheOREM 9. For any $i>2$, there exists a valuation $w$ in $L_{i}$ such that for the relation $\approx_{w}^{i}$ rules (Ad), (Or) do not hold.

Proof. We will prove the theorem for $i=3$. Valuation $w$ defined below can be extended in a natural way to any language $L_{k}$, for $k>3$.

The tabular below determine a probability distribution. The last column set the probabilities of terms listed in a given line - taken in a conjunction they form state descriptions that sum up vertically to 1 . For example 0,4 in the third line means that $w(p \wedge \neg q \wedge r)=0,4$.

To calculate any conjunction of literals we just add all the numbers in the last column of all the lines containing all literals of a given conjunction. For example to calculate $w(p \wedge q)$ we take line 1 and line 5 and then add respective numbers: $0+0,1=0,1$.

According to the table 1 we have in particular: $w(p \wedge q)=0,2 ; w(p \wedge$ $r)=0,3 ; w(q \wedge r)=0,3 ; w(p)=0,5 ; w(q)=0,5 ; w(r)=0,6$.

(Ad) We will show that (Ad) fails under $w$. Thus we have $w(p \wedge r)=0,3 \geqslant$ $0,3=0,5 \cdot 0,6=w(p) \cdot w(r)$ and $w(q \wedge r)=0,3 \geqslant 0,3=0,5 \cdot 0,6=$ $w(q) \cdot w(r)$. Hence $p \approx r$ and $q \approx r$, by Theorem 4 . On the other hand $w(p \wedge q \wedge r)=0<0,12=0,2 \cdot 0,6=0,3=w(p \wedge q) \cdot w(r)$. Hence $p \wedge q \not \notin r$, by Theorem 4 . 


\begin{tabular}{|c|c|c|c|c|}
\hline$p$ & $q$ & $r$ & $=$ & 0 \\
$\neg p$ & $q$ & $r$ & $=$ & 0,3 \\
$p$ & $\neg q$ & $r$ & $=$ & 0,3 \\
$\neg p$ & $\neg q$ & $r$ & $=$ & 0 \\
$p$ & $q$ & $\neg r$ & $=$ & 0,2 \\
$\neg p$ & $q$ & $\neg r$ & $=$ & 0 \\
$p$ & $\neg q$ & $\neg r$ & $=$ & 0 \\
$\neg p$ & $\neg q$ & $\neg r$ & $=$ & 0,2 \\
\hline
\end{tabular}

Table 1. Values of state descriptions

(Or) Since $w(r \wedge \neg q)=w(\neg q \wedge r)=0,3$, by (W8), and $w(r)=0,6$; $w(\neg q)=0,5$; so $w(r \wedge \neg q) \geqslant 0,3 \geqslant 0,6 \cdot 0,5=w(r) \cdot w(\neg q)=0,3$; and hence $r \approx \neg q$, by Theorem 4. Since $w(p \wedge \neg q)=0,3$ and $w(p)=0,5$; $w(\neg q)=0,5$; so $w(p \wedge \neg q) \geqslant 0,5 \cdot 0,5=w(p) \cdot w(\neg q)=0,25$; and hence $p \approx \neg q$.

Now, $(p \vee r) \wedge \neg q$ is logically equivalent to $(*):(p \wedge \neg q \wedge r) \vee(\neg p \wedge \neg q \wedge$ $r) \vee(p \wedge \neg q \wedge \neg r)$. However, $w((*))=0,3+0+0=0,3$; and by (W8), $w((p \vee r) \wedge \neg q)=0,3$. At the same time $w(p \vee r)=w(p)+w(r)-w(p \wedge r)=$ $0,5+0,6-0,3=0,8$, by (W9). Thus $w(p \vee r) \cdot w(\neg q)=0,8 \cdot 0,5=0,4$ and $w((p \vee r) \wedge \neg q)<w(p \vee r) \cdot w(\neg q)$. As a consequence $p \vee r \not \notin \neg q$, by Theorem 4 .

Proposition 10. (i) If a valuation $w$ in $L_{1}$ has at least one value different from 0 and 1 , then $A \approx_{w}^{1} B$ if and only if $A \models B$ or $B \models A$.

(ii) If $w$ has only values 0 and 1 , then $A \approx_{w}^{1} B$, for any $A, B$.

Proof. (i) Suppose that $w(p)=r \notin\{0,1\}$. By the rule (Cl) i (Sym) if $A \models B$ or $B \models A$, then $A \approx_{w}^{1} B$. Let us suppose that for some $w$ : $A \approx_{w}^{1} B$, but neither $A \models B$ nor $B \models A$. Since we have only two state descriptions: $p$ and $\neg p$, so we can assume that $A$ and $B$ may be only of forms logically equivalent to sentences: $p \wedge \neg p, p, \neg p, p \vee \neg p$. The only such a pair $A, B$ that $A \not \forall B$ and $B \not \forall A$ is $p$ and $\neg p$. However, $w(p \wedge \neg p)=0<r \cdot(1-r)=w(p) \cdot w(\neg p)-$ a contradiction.

(ii) Obviously $w(p)=0$ or $w(\neg p)=0$. If $w(\neg p)=0$, then $w(p \wedge \neg p)=$ $0 \geq 0=w(p) \cdot w(\neg p)$. Hence $p \approx_{w}^{1} \neg p$. Similarly we check other cases. 
We will modify here the rules investigated in this section to adopt them to the relation of support. Intuitively, the first idea of such a modification is to limit the scope of rules. We will call the rules modified and add suffix -m (as modified) to their original names.

(RW-m)

$$
\frac{\models A \rightarrow B, \quad C \models A}{C \approx B}
$$

(Cut-m)

$$
\frac{A \wedge B \approx C, \quad A \models B}{A \approx C}
$$

$$
\frac{A \approx C, \quad B \models C}{A \wedge B \approx C}
$$

(Mon-m)

$$
\frac{A \models C}{A \wedge B \approx C}
$$

(CM-m)

$$
\frac{A \models B, \quad A \approx C}{A \wedge B \approx C}
$$

(Eq-m)

$$
\begin{array}{ll}
A \approx B, & B \models A, \quad A \models C \\
B \approx C &
\end{array}
$$

(Or-m)

$\frac{A \models \neg B, \quad A \approx C, \quad B \approx C}{A \vee B \approx C}$

(T-m)

$$
\frac{A \models B, \quad B \models C}{A \approx C}
$$

Theorem 11. The rules (RW-m), (Cut-m), (Mon-m), (CM-m), (Eq-m), (T-m) hold for any valuation $w$.

Proof. Let us consider for example the few following cases.

(Cut-m) Let us suppose that $A \wedge B \approx C$ and $A \models B$. Then from (W8) $w(A)=w(A \wedge B)$. We have $w(A \wedge C) \geq w(A \wedge B \wedge C) \geq w(A \wedge B) \cdot w(C)=$ $w(A) \cdot w(C)$. In consequence $A \approx C$.

(CM-m) Let us suppose that $A \models B$ and $A \approx C$. Then from (W8) $w(A)=$ $w(A \wedge B)$ and $w(A \wedge C)=w(A \wedge B \wedge C)$. We have $w(A \wedge B \wedge C)=$ $w(A \wedge C) \geq w(A) \cdot w(C)=w(A \wedge B) \cdot w(C)$. In consequence $A \wedge B \approx C$. 
(Or-m) Let us suppose that $A \models \neg B, A \approx C$ and $B \approx C$. Then of course $A \wedge C \models \neg(B \wedge C)$. In consequence - by (W8) and (W4) - we have $w((A \vee B) \wedge C)=w((A \wedge C) \vee(B \wedge C))=w(A \wedge C)+w(B \wedge C) \geq$ $w(A) \cdot w(C)+w(B) \cdot w(C)=(w(A)+w(B)) \cdot w(C)=w(A \vee B) \cdot w(C)$. Hence $A \vee B \approx C$.

The remaining cases are provable by properties of classical consequence relation $\models$. Some of them, like for example (Eq-m) or (T-m), are particular instances of $(\mathrm{Cl})$.

Proposition 12. For any $n$ there exists only finitely many distinct relations $\approx_{w}^{n}$. The number of distinct relations is less than $2^{2^{4 n}}$.

Proof. Let for $A \in L_{n}[A]$ denote set $\{B: B \models A$ and $A \models B\}$ and let $l_{n}$ denote such a set that any set $l_{n} \cap[A]$ has exactly one element. The set $l_{n}$ is finite, it has $2^{2 n}$ elements. Given a valuation $w$, from (LLE) and (RLE): if $A_{1} \models A_{2}, B_{1} \models B_{2}$, then $A_{1} \approx_{w}^{n} B_{1}$ iff $A_{2} \approx_{w}^{n} B_{2}$.

As a consequence any relation $\approx_{w}^{n}$ is uniquely determined by its behavior on the set $l_{n}$. Hence the upper limit of the number of relations $\approx_{w}^{n}$ is the number of subsets of $l_{n} \times l_{n}$.

Valuations $w_{1}$ and $w_{2}$ are equivalent (symbolically: $w_{1} \doteqdot w_{2}$ ) iff $\approx_{w_{1}}=\approx_{w_{2}}$. The problem of a useful characterization of the relation $\doteqdot$ we leave to a future examination.

\section{Appendix: Two demonstrative examples.}

The conditions of monotonicity (Mon) and idempotency (Cut) are very natural and important from a point of view of the theory of logical consequence. However, we saw they fail in the context of support relation, which is the case since probability can change in respect to appearance of a new information. For this reason we would like to present two examples, which are demonstrative illustrations of such failures.

The failure of (Mon). A pot with 10 balls is given. Each ball has a number 1 or 2 and is either red or green. Among them there are: three red balls with 1 , two green balls with with 1 , two red balls with 2 , and three green balls with 2. We choose a ball randomly. Suppose it is red. Intuitively we expect that there is rather 1 than 2 on this ball, since there are more red balls with 1 than with 2 . We will construct a logic which confirm and formalize this intuition. 
Let $x \in\{$ red, green, 1,2$\}$. Symbol $x$ is a shorthand for a sentence: 'A ball of the color/number $x$ is randomly chosen'. Let $L_{4}$ denote a propositional language with four variables. ${ }^{2}$ Each variable of $L_{4}$ corresponds to one of the sentences listed above. The valuation $w$ will be defined as a probability - for any sentence $A, w(A)$ is a probability of an event described by $A$. $w_{A}(B)$ denotes the conditional probability based on $w: w_{A}(B)=\frac{w(A \wedge B)}{w(A)}$, provided that $w(A) \neq 0$. In particular we have:

a) $w(1)=1 / 2$, since half of balls is with 1 , and the rest with 2

b) $w(1 \wedge(\operatorname{red} \wedge 1))=w($ red $\wedge 1)=3 / 10$, since three of ten balls are red and 1

c) $w(1 \wedge$ green $)=2 / 10$, since two of ten balls are 1 and green

d) $w((1 \wedge$ green $) \wedge($ red $\wedge 1))=w($ green $\wedge$ red $)=0$, since no ball is red and green.

We have $w_{1}(\operatorname{red} \wedge 1)=\frac{w(1 \wedge(\operatorname{red} \wedge 1))}{w(1)}=\frac{3 / 10}{1 / 2}=3 / 5 \geqslant 3 / 10=w(\operatorname{red} \wedge 1)$. Hence $1 \approx_{w}$ red $\wedge 1$. If we add new premise green to 1 , then relation of support will fail. We have $1 \wedge$ green $\not \psi_{w}$ red $\wedge 1$, because $w_{1 \wedge \text { green }}($ red $\wedge 1)=$ $\frac{w((1 \wedge \text { green }) \wedge(\text { red } \wedge 1))}{w(1 \wedge \text { green })}=\frac{0}{2 / 10}=0<3 / 10=w($ red $\wedge 1)$. Therefore $1 \approx_{w}$ red $\wedge 1$ and $1 \wedge$ green $\mathscr{L}_{w}$ red $\wedge 1$, so (Mon) fails.

The failure of (Cut). Let us consider the following story. Somebody has been murdered. There are four suspects: $a, b, c, d$. Suppose that only one person is committed the murder. Initially the four suspects are equally suspected and there is no reason to suspect anybody else. Then two levels of evidence arrive.

EVIDENCE 1. Suspect $d$, and only $d$, becomes more suspected. The probability that he is the murder increases from $1 / 4$ to $1 / 3$. Therefore probability of being the murder for $a, b, c$ is now $2 / 3 \cdot 1 / 3=2 / 9$.

Evidence 2. Suspects $b$ and $c$ are not longer suspected. For example they can have a very strong alibi. So, when this evidence arrives only $a$ and $d$ remain suspected. ${ }^{3}$

\footnotetext{
${ }^{2}$ It is obvious that any of the atomic sentences could be represented as a negation of some sentence, for example it could be: green $\leftrightarrow \neg$ red and $2 \leftrightarrow \neg 1$. So, we could reduce the language to two variables. However, for the simplicity of considerations we assume four atomic sentences.

${ }^{3}$ This story is a modification of the story considered in J. Pearl [89] and [2002].
} 
Let $x \in\{a, b, c, d\}$ be a shorthand for ' $x$ is guilty'. Let $e_{1}$ and $e_{2}$ denote respectively the content of level of evidence 1 and of level of evidence 2. Let $L_{6}$ denotes the propositional language with 6 sentential variables. While interpreting the language we link the variables with sentences from the story. It will not cause misunderstanding if we just call the variables respectively $a, b, c, d, e_{1}, e_{2}$. Let $w$ denotes a valuation which to any sentences of $L_{5}$ ascribe its probability respectively to the story above. In particular we get:

a) for $x \in\{a, b, c, d\} w(x)=1 / 4$, since there are four suspects

b) $w\left(e_{1}\right)=w\left(e_{1}\right)=1 / 2$, since it can be the case what evidences say or may not be

c) $w_{e_{1}}(d)=1 / 3$, since $e_{1}$ states that probability of $d$ increases to $1 / 3$

d) $w_{e_{1}}(a)=2 / 9$, since $e_{1}$ states that only probability of $d$ increases to $1 / 3$

e) $w_{e_{1}}\left(e_{2}\right) \geqslant w\left(e_{2}\right)$, since $e_{1}$ does not decrease a probability of $e_{2}$.

Let us calculate a conditional probability $w_{e_{1} \wedge e_{2}}(a)$. According to evidence 1 probability of $a, b, c, d$ is respectively equal to $2 / 9,2 / 9,2 / 9,1 / 3$. So $w_{e_{1}}(b)+w_{e_{1}}(c)=4 / 9$. When evidence 2 arrives probabilities of $b$ and $c$ go down to 0 and probabilities of $a$ and $d$ go up. The prior probability of $b$ and $c$ should be divided among $a$ and $d$ in such a way that a proportion of probabilities of $a$ and $d$ is preserved. We have then to find a number $0 \leqslant x \leqslant 1$ such that:

$$
\frac{w_{e_{1}}(a)}{w_{e_{1}}(d)}=\frac{2 / 9}{1 / 3}=\frac{x}{1-x}
$$

Therefore $x=2 / 5$ and hence:

$$
w_{e_{1} \wedge e_{2}}(a)=2 / 9+2 / 5 \cdot 4 / 9=2 / 5>1 / 4=w(a)
$$

In consequence $e_{1} \wedge e_{2} \approx_{w} a$. On the other hand, $w_{e_{1}}(a)=2 / 9<1 / 4=$ $w(a)$ and hence $e_{1} \not \mathcal{L}_{w} a$. In result we get $e_{1} \wedge e_{2} \approx_{w} a, e_{1} \approx_{w} e_{2}$ and $e_{1} \not z_{w} a$. Therefore (Cut) fails.

ACKnowledgements. The authors are very indebted to David Makinson for very helpful comments on draft version of this paper. 


\section{References}

[1] R. Carnap, Logical Foundation of Probability, Routledge and Kegan Paul, London (1951).

[2] C. Howson, P. Urbach Scientific reasoning: the Bayesian approach, La Salle, Illinois (1990).

[3] A. N. Kolmogorov, Foundations of the theory of probability second english edition, Chelsea Publishing Company, New York (1956).

[4] S. Kraus, D. Lehmann and M. Magidor, Nonmonotonic Reasoning, Preferential Models and Cumulative Logics, Artificial Intelligence 44 (1990), pp. 167-207.

[5] T. Kuipers, Studies in Inductive Probability and Rational Expectation, Reidel, Dordrecht (1978).

[6] T. Kuipers, From Instrumentalism to Constructive Realism, Synthese Library 287, Kluwer Academic Press, Dordrecht (2000).

[7] D. Makinson, Bridges from Classical to Nonmonotonic Logic, Texts in Computing, Kings College, London (2005).

[8] J. Pearl, Probabilistic Reasoning in Intelligent Systems, Morgan Kaufman, San Mateo, CA (1978).

[9] J. Pearl, On Two Pseudo-Paradoxes in Bayesian Analysis, Annals of Mathematics and Artificial Intelligence 32(2001), pp. 171-177.

[10] K. Popper, The Logic of Scientific Discovery, revised edition, Hutchinson, London (1968).

Institute of Philosophy and Sociology

Polish Academy of Sciences

e-mail: jacek.malinowski@studialogica.org

Department of Logic

Nicolaus Copernicus University in Toruń

e-mail: jarmuzek@umk.pl

e-mail: matklon@doktorant.umk.pl 